Supporting Information for

\title{
Neural Network Sampling of the Free Energy Landscape for Nitrogen Dissociation on Ruthenium
}

Elizabeth M. Y. Lee, ${ }^{1, \ddagger}$ Thomas Ludwig, ${ }^{2,3, \neq}$ Boyuan Yu, ${ }^{1}$ Aayush R. Singh,,${ }^{2, t}$

François Gygi, ${ }^{4}$ Jens K. Nørskov, ${ }^{2,3,5 *}$ and Juan J. de Pablo ${ }^{1,6 *}$

${ }^{1}$ Pritzker School of Molecular Engineering, The University of Chicago, Chicago, Illinois 60637, USA

${ }^{2}$ SUNCAT Center for Interface Science and Catalysis, Department of Chemical Engineering, Stanford University, Stanford, California 94305, United States

${ }^{3}$ SUNCAT Center for Interface Science and Catalysis, SLAC National Accelerator Laboratory, 2575 Sand Hill Road, Menlo Park, California 94025, United States

${ }^{4}$ Department of Computer Science, University of California, Davis, California 95616, USA

${ }^{5}$ Department of Physics, Technical University of Denmark, Lyngby 2800, Denmark

${ }^{6}$ Argonne National Laboratory, 9700 Cass Avenue, Lemont, IL 60439, USA.

¥These authors contributed equally.

†Present address: Dow Performance Silicones, 2651 Salzburg Road, Midland, MI, 48686

*corresponding authors: jkno@dtu.dk, depablo@uchicago.edu 


\section{Density Functional Theory (DFT) Calculations}

Electronic structure computations and molecular dynamics were carried out at the level of DFT using the PBE exchange-correlation functional ${ }^{1}$, with SG15 Optimized NormConserving pseudopotential ${ }^{2,3}$, as implemented in the Qbox code ${ }^{1}$. A plane-wave basis set expanded to an energy cutoff 50 Ry was used to represent the valence electrons, and a Fermi smearing with electronic temperature of $1090 \mathrm{~K}$.

The bulk lattice constant was determined by optimizing the lattice constant of a bulk HCP unit cell. The unit cell used 4 Ru atoms in an orthogonal cell representing an HCP bulk structure. The atomic forces and unit cell stress were minimized using a conjugate-gradient algorithm. The atoms and length of lattice vectors were allowed to change to minimize the forces and stress, whereas the cell angles were held constant. Lattice constants for the HCP crystal structure of $a=2.725 \AA$ and $c=4.290 \AA$ were obtained, which are in good agreement with experimental data $(a=2.706 \AA$ and $c=4.282$ $\AA)$.

For all geometry optimization, transition state search calculations, and molecular dynamics calculations, the bottom surface layer was frozen at the bulk value from the lattice constant optimization, while the top layer and all additional atoms (adsorbates) were allowed to move. For geometry optimizations, the conjugate-gradient algorithm was used to minimize the energy and forces, with final states having a maximum force less than $0.05 \mathrm{eV} / \AA$.

\subsection{Benchmark Studies for DFT-PBE}

To test convergence, the primary quantity of importance was the surface reaction energy, or the difference between energies of the $\mathrm{N}_{2}{ }^{*}$ and $2 \mathrm{~N}^{*}$ states. Therefore, we benchmarked this reaction energy as a function of various model parameters (plane wave cutoff, number of k-points, number of Ru layers, and vacuum layer thickness). For each set of model parameter values, we optimized the bulk lattice constant and reported the surface reaction energy. The results of the convergence study of DFT parameters are summarized in Figure S1.

Since enhanced sampling simulations with ab initio MD (AIMD) can call upon a large number of DFT calculations to converge sampling statistics, we chose the following DFT parameters to strike a balance between accuracy and performance: a plane wave cutoff of $50 \mathrm{Ry}$, a $2 \times 2 \times 1 \mathrm{k}$-point mesh, a two-layer Ru slab, and a vacuum layer thickness of $10.1 \AA$.

\section{Transition State Search}

Conventionally, the Climbing Image Nudged Elastic Band $(\mathrm{Cl}-\mathrm{NEB})^{4}$ is used to determine transition states and associated activation energies in heterogeneous catalysis. But in simple reactions such as diatomic dissociation/association reactions, the Fixed Bond Length (FBL) method has been successfully used in the past. ${ }^{5}$

To benchmark the FBL method used in this work, we directly compare the results from FBL and Cl-NEB for finding the transition state for the $\mathrm{N}_{2}{ }^{*} \rightarrow 2 \mathrm{~N}^{*}$ reaction. The calculations were carried out using the Quantum Espresso software package ${ }^{6}$, with the CI-NEB method implemented in the Atomic Simulation Environment (ASE) ${ }^{7}$. We found almost exact agreement in resulting geometries and energies of transition states from the two methods (e.g., within $0.001 \mathrm{eV}$ in DFT energy). This result indicates that for the 
reaction studied in this work, the FBL method finds essentially the same transition state, and thereby, the activation energy, as the Cl-NEB method.

In FBL calculations, transition states were identified by first plotting the potential energy (the Kohn-Sham energy from DFT) versus the $\mathrm{N}-\mathrm{N}$ bond length and then performing normal mode calculations. We verified that the transition state was indeed a saddle point of the potential energy surface by finding a single imaginary mode and all other modes to be real. The structure of each configuration (the two nitrogen atoms and the mobile surface atoms in the top layer) was optimized using energy minimization while constraining the N-N bond length to its fixed value. In Figures S2 and S3, we show a summary of FBL calculations resulting in two lowest energy transition state configurations. Only the lowest-energy transition state, shown in Figure S2, was reported as the transition state of the potential energy surface.

\section{Free Energy Calculations using Harmonic Approximation (HA)}

Critical points on the potential energy surface (found using energy minimization or $\mathrm{FBL}$ ) were used to perform free energy calculations using harmonic approximation (HA). For each critical state, normal modes were determined by diagonalizing the massweighted Hessian matrix or the force constant matrix (i.e., the matrix of second derivatives of the PES with respect to the mass-weighted atomic coordinates), constructed via displacements of the atoms considered. ${ }^{8}$ In the SC-HA and MC-HA methods, only the adsorbate (nitrogen) atoms are considered in the Hessian matrix (the surface atoms are of course included in the energy and force evaluations, but only the adsorbate atoms are displaced for the finite difference calculations). In the MCS-HA method, the normal mode analysis was performed using adsorbate atoms and the metal atoms in the top layer of the metal surface (see Section S3.3 for further discussion on surface vibrations). The thermochemistry module in $\mathrm{ASE}^{7}$ was used to compute the free energy, enthalpy, and entropy for each state.

\subsection{Comparison of Free Energies from Classical and Quantum Harmonic Oscillators}

One possible source of disagreement between HA and CFF-AIMD could be the discrepancy between a quantum and classical treatment of the nuclei. The harmonic approach uses a quantum mechanical treatment, which includes, for example, zero point energy (ZPE). The relevant equations for HA are well known and are already implemented in the ASE thermochemistry module, but are provided here for the sake of comparison:

$$
\begin{gathered}
S_{v i b}=k_{B} \sum_{i}\left[\frac{\epsilon_{i}}{k_{B} T\left(e^{\frac{\epsilon_{i}}{k_{B} T}}-1\right)}-\ln \left(1-e^{-\frac{\epsilon_{i}}{k_{B} T}}\right)\right] \\
\int_{0}^{T} C_{V, v i b} d T=\sum_{i} \frac{\epsilon_{i}}{e^{\frac{\epsilon_{i}}{k_{B} T}}-1} \\
E_{Z P E}=\sum_{i} \frac{1}{2} \epsilon_{i}
\end{gathered}
$$




$$
G_{\text {harmonic }}=E_{\text {electronic }}+E_{Z P E}+\int_{0}^{T} C_{V, v i b} d T-T S_{v i b}
$$

We also calculated the free energy for the classical harmonic oscillator. The equations used are shown here:

$$
\begin{gathered}
S_{v i b}=k_{B} \sum_{i}\left[-\ln \left(\frac{\epsilon_{i}}{k_{B} T}\right)+1\right] \\
\int_{0}^{T} C_{V, v i b} d T=\sum_{i} k_{B} T \\
G_{\text {classical }}=E_{\text {electronic }}+\int_{0}^{T} C_{V, v i b} d T-T S_{v i b} \quad(\mathrm{~S} 2),
\end{gathered}
$$

In above equations, the indices $i$ indicate a sum over all the vibrational modes considered. The direct comparison of the results for these models are displayed in Table S1.

The differences are relatively small even at low temperature due to a relatively small ZPE effect in this reaction. At the temperatures studied in this work $(700-1400 \mathrm{~K})$, the differences are even smaller (on the order of 0.01 to $0.02 \mathrm{eV}$ ). There is a good agreement in HA results using quantum and classical harmonic approximation for this reaction, providing evidence that quantum effects such as ZPE do not substantially affect the comparisons between CFF-AIMD and HA made in this work.

\subsection{Configurational Multiplicities in MC-HA and MCS-HA Calculations}

For all of the states considered in this study, there are multiple equivalent atomic configurations within the simulation supercell. The number of such equivalent configurations is called the "degeneracy" or configurational multiplicity. Degeneracies are additional contributions to the total entropy through configurational entropy. Thus we enumerated all of these configurations for relevant states, as shown in Figure S4.

In order to compare CFF-AIMD and HA, we consider both the entropy from the degeneracy of each configuration $\left(\mathrm{N}_{2}{ }^{*}, \mathrm{TS}\right.$, and $\left.2 \mathrm{~N}^{*}\right)$ and a Boltzmann distribution of $2 \mathrm{~N}^{*}$ configurations observed within a radius of $0.05 \AA$ of the $2 \mathrm{~N}^{*}$ minimum in the $(r, Z)$-collective variable space: HCP-HCP $60^{\circ}$, HCP-HCP $0^{\circ}$, FCC-FCC 60', and FCC-FCC $0^{\circ}$ (see Figure S11).

\subsection{Discussion on surface phonon modes in MCS-HA}

The surface unit cell is a (2x2) supercell of the primitive cell of the $\mathrm{Ru}(0001)$ surface. Therefore, the MCS-HA method considers surface phonons at the $\bar{\Gamma}$ point and the boundary points of the surface Brillouin zone of the primitive cell. Previous quasiclassical trajectory studies have indicated that the coupling between adsorbate vibrational modes and surface phonons can affect the dissociative sticking probability of $\mathrm{N}_{2}$ on $\mathrm{Ru}(0001)^{9}$, suggesting other surface phonon modes may affect reaction energies and barriers.

\section{Anharmonicity Effect}

To investigate whether there is a strong anharmonicity for a given state, we computed a potential energy curve as a function of atomic displacements in the direction of the eigenvector having the smallest eigenvalue (the lowest normal mode frequency) of the Hessian matrix. In Figure S5, we show local potential energy profiles for $\mathrm{N}_{2}{ }^{*}$, TS, and 
$2 \mathrm{~N}^{*}$ from single-point DFT calculations and the harmonic approximations. The lowestenergy vibrational modes for these states appear to be mostly harmonic, especially for $2 \mathrm{~N}^{*}$, when the energy above the minimum is less than $0.2 \mathrm{eV}$. The largest degree of anharmonicity is seen for the $\mathrm{N}_{2}{ }^{*}$ state.

\section{Enhanced Sampling Simulations}

\subsection{Combined Force Frequency (CFF) Method}

In the original CFF method ${ }^{10}$, the system was not biased during the initial sweep of CFF (prior to the onset of neural network derived forces), and the method was implemented for classical MD with a single replica. This CFF method has now been extended to increase the sampling efficiency and the range of applicable systems. New features include biased AIMD simulation by coupling SSAGES ${ }^{11}$ and Qbox $^{1}$, a new initialization method using the ABF algorithm in the initial sweep to bias the simulation, multi-walker sampling for parallel computing, and non-rectangular CV gridding to avoid unphysical regions of the phase space. Our CFF method is now available in the latest version of the SSAGES software package (v0.9).

CFF proceeds similarly to the ABF method ${ }^{12}$ but uses Bayesian self-regularizing artificial neural networks (BRANNs) to represent free energies and biasing forces that are continuous over the CV space.

In CFF, two neural networks are defined to represent the bias: one trained from frequencies of state visits ("freq"), and the other trained from both the frequencies and mean forces ("force"). The mean force at the CV coordinate $\xi$ is denoted by $\tilde{\mathbf{F}}_{\xi}$ and is expressed as follows ${ }^{12}$ :

$$
\tilde{\mathbf{F}}_{\xi}=\nabla_{\xi} G(\xi)=-\langle\mathbf{F}\rangle_{\xi}=-\left\langle\frac{\partial}{\partial t} \mathbf{W}^{\mathrm{T}} \mathbf{p}\right\rangle_{\xi}
$$

where $G$ is the free energy; $\xi$ is a vector of $\mathrm{CVs} ; \nabla_{\xi} G$ is the mean force or the gradient of the free energy with respect to the $\mathrm{CVs}$; $\mathbf{F}$ is the instantaneous force acting on $\mathrm{CVs}$; and the angle brackets as in the term $\langle\mathbf{F}\rangle_{\xi}$ denote the running time average (for example, of the instantaneous force) at the CV coordinate $\xi$. The instantaneous force is defined as the time derivative of $\mathbf{W}^{\mathrm{T}} \mathbf{p}$ where $\mathbf{p}$ is the vector of atomic momenta, and $\mathbf{W}$ is the right pseudoinverse of $\mathbf{J}$, the Jacobian matrix of $\mathrm{CVs}$ with respect to the atomic coordinates, i.e., $\mathbf{W}=\mathbf{J}^{\mathrm{T}}\left(\mathbf{J J}^{\mathrm{T}}\right)^{-1} .13$

Each neural network is optimized by minimizing the mean squared error (MSE) of its loss function with respect to the network weights. The loss function of the frequencybased BRANN is given by

$$
\mathrm{MSE}_{\text {freq }}=\beta_{\text {freq }} \sum_{i}\left(\tilde{G}_{i}-Q_{\text {freq }, i}\right)^{2}+\alpha_{\text {freq }} \sum_{j} w_{\text {freq }, j}{ }^{2}
$$

where the index $i$ in this equation runs over all bins in the CV space, and the index $j$ runs over all weights in the network, $\left\{w_{\text {freq }, j}\right\}$. This loss function consists of the network targets, $\left\{\tilde{A}_{i}\right\}$, which are instantaneous free energies calculated from visit frequencies; the network predictions for free energies, $\left\{Q_{\text {freq }, i}\right\}$; and regularization parameters, $a_{\text {freq }}$ and $\beta_{\text {freq }}$, whose ratio, $\gamma_{\text {freq }}=\alpha_{\text {freq }} / \beta_{\text {freq }}$, defines the effective number of parameters in the network. ${ }^{14}$ Similarly, the loss function of the force-based BRANN is derived as 


$$
\mathrm{MSE}_{\mathrm{force}}=\beta_{\text {force }} \sum_{i}\left[\left(\tilde{G}_{i}-Q_{\text {force }, i}\right)^{2}+\left(\tilde{F}_{i}-\dot{Q}_{\text {force }, i}\right)^{2}\right]+\alpha_{\text {force }} \sum_{j} w_{\text {force }, j}{ }^{2}
$$

where $\left\{\tilde{F}_{i}\right\}$, the mean forces defined in Eq. 3 are the network targets, and $\left\{\dot{Q}_{\text {force }, i}\right\}$ are the derivatives of the network function with respect to the network inputs (the CVs). In CFF, the biasing force is a weighted average of the derivatives of both network functions:

$$
\mathbf{F}_{\text {bias }}(\xi)=\gamma \dot{Q}_{\text {freq }}(\xi)+(\gamma-1) \dot{Q}_{\text {force }}(\xi)
$$

where $\gamma \in[0,1]$ is the relative complexity of the two networks, i.e., $\gamma=\gamma_{\text {freq }} /\left(\gamma_{\text {freq }}+\gamma_{\text {force }}\right)$, which is dynamically updated during a CFF simulation. ${ }^{10}$

CFF estimates the free energy by determining the force that a molecular system experiences along collective variable(s) and adds an opposing force (the "bias" represented by neural networks in Eq. S6) in an iterative fashion, such that the total force is zero.

\subsection{Neural Network Sensitivity Analysis}

We investigate the sensitivity of CFF performance to the neural network architectures using alanine dipeptide in explicit water, a prototype system for testing and benchmarking enhanced sampling methods ${ }^{10,13,14}$. The system consists of alanine dipeptide and 880 water molecules. 3D periodic boundary conditions were imposed on an orthorhombic unit cell of approximately $3 \mathrm{~nm} \times 3 \mathrm{~nm} \times 3 \mathrm{~nm}$. CFF-MD simulations of alanine dipeptide were carried out using Gromacs software package ${ }^{15}$ coupled to SSAGES with Amber ff99SB ${ }^{16}$ for the peptide and TIP3P ${ }^{17}$ for water using a single walker. Dynamics were simulated at the NPT ensemble using a $1 \mathrm{fs}$ timestep with temperature and pressure controlled by the stochastic velocity rescaling thermostat at $298.15 \mathrm{~K}$ and the Parrinello-Rahman barostat ${ }^{18}$ at 1 bar, respectively. CVs were the two torsional angles $\varphi$ and $\psi$ of alanine dipeptide. The 2D CV grid consisted of 30 by 30 points and was bounded by $\varphi \in[-\pi, \pi]$ and $\psi \in[-\pi, \pi]$. For the BRANN architecture, two hidden layers ( $N$ by $M$ nodes) were used, and neural networks were trained after every 10 ps.

The root mean squared error (RMSE) of PMFs overtime for various network sizes is shown in Figure S6. Except for the smallest network used in this study (2 hidden layers, 6 by 2 nodes), all PMFs converged to within $\sim 5 \mathrm{~kJ} / \mathrm{mol}$ or $\sim 2 \mathrm{k}_{\mathrm{B}} T$ within $\sim 2 \mathrm{~ns}$. Therefore, the CFF algorithm appears to be largely insensitive to the network architecture as long as the network consists of two hidden layers (for two or more CVs) with more than 8 and 4 nodes in the first and the second hidden layers, respectively. Ordinarily, increasing the size of the NN architecture would lead to the risk of over-fitting, but the Bayesian regularization method used in our method greatly reduces this risk, as discussed in a previous work utilizing the Bayesian regularization ${ }^{14}$.

\subsection{Discussion on sampling using ABF-AIMD}

The ABF-AIMD method failed to sample regions proximal to the $\mathrm{N}_{2}{ }^{*}$ state because the energy surface in the $\mathrm{N}_{2}{ }^{*}$ region changes more rapidly (especially along the " $r$ " coordinate), compared to the energy landscape at the vicinity of the $2 \mathrm{~N}^{*}$ state (see Figure S9A). In ABF-AIMD simulations, when the system was initialized at the $\mathrm{N}_{2}{ }^{*}$ minimum, it was unable to overcome steep barriers away from the minimum to visit the neighboring regions. Hence, biasing forces in those regions were zero (see also Figure S9B). On the 
other hand, the energy well in the $2 \mathrm{~N}^{*}$ region is broader and less steep; the system initialized in the $2 \mathrm{~N}^{*}$ region escaped the $2 \mathrm{~N}^{*}$ minimum and visited neighboring regions.

\subsection{Calculation of Free Energy and Minimum Free Energy Paths}

In CFF-AIMD simulations, BRANNs used two hidden layers (14 by 10 nodes). We chose a grid spacing of $\Delta=0.1$ Bohr along each direction in the two-dimensional CV grid bounded by $r \in[1.6,6.8]$ Bohr and $Z \in[1.6,6.0]$ Bohr (see Figure S7) to compute mean forces (Eq. S3) and record visit frequencies. In the first 4 ps, the CFF algorithm proceeded in the same manner as the ABF method where the biasing force is defined as a negative of the mean force. Afterward, the biasing force in CFF was switched to the neural network representation in Eq. S6. Neural networks were trained using newly updated mean forces and frequencies of visits after every $2 \mathrm{ps}$. The simulation was terminated when every CV bin was filled (more than 30 counts for each bin). After the simulation was stopped, the root-mean-square (RMS) error in free energy (calculated with respect to the longest CFFAIMD simulation run in Table S2) was less than $0.1 \mathrm{eV}$. Multiple walkers (13 to 25 on average) were employed to increase the CFF sampling efficiency. The PMF was computed by integrating mean forces over the 2D-CV grid to evaluate the performance of neural networks by comparing two similar methods with and without neural network (CFF and ABF). For speed comparison between ABF and CFF, we used 16 walkers throughout the simulation. Summary of all CFF-AIMD simulations are reported in Table S2.

At each temperature, CFF-AIMD results were averaged over three independent enhanced sampling simulations to compute the standard deviation. Mean free energy paths (MFEPs) from CFF-AIMD simulations were directly calculated from results of CFFAIMD by first fitting the average 2D-PMF to BRANNs. This fitting procedure enables a smooth interpolation of free energy from a discretized two-dimensional CV grid. We used MATLAB 2019a Deep Learning Toolbox to train BRANNs having one hidden layer with 100 nodes. BRANNs are optimized using convergence tolerances of $10^{-3}$ and $10^{-5}$ for the objective function and the gradient, respectively. BRANNs were used as an energy function to locate minima $\left(\mathrm{N}_{2}{ }^{*}\right.$ and $\left.2 \mathrm{~N}^{*}\right)$, calculate the MFEP using $\mathrm{NEB}^{19}$, and find the transition state along the MFEP. Final free energy surfaces after averaging three independent runs at each temperature are shown in Figure S8.

\subsection{Calculation of Entropy and Enthalpy}

The entropy part is calculated as, $-T \Delta S(q)=\Delta G(q)-\left\langle\Delta E_{P}(q)\right\rangle-P \Delta \mathrm{V}$, where $\Delta G(q)$ is the free energy computed from CFF-AIMD at $q$, a point in the CV space. The pressurevolume term, $P \Delta \mathrm{V}$, is negligeable for the surface reaction. Therefore, the enthalpy part, $\Delta H(q)$, is equal to $\left\langle\Delta E_{P}(q)\right\rangle$, the average DFT potential energy at point $q$, calculated from CFF-AIMD simulations.

\subsection{Referencing the Free Energy to the Nitrogen Gas Phase}

While it is possible within the enhanced sampling framework to directly compute PMF for the molecular dissociation process starting from the gas phase, this would require using a larger supercell size (e.g., twice as long in the z-direction) than what was used, which was not practically feasible based on our available computational resources. Therefore, we used the ideal gas and harmonic approximations to change the reference 
state of FES calculated from CFF-AIMD from the $2 \mathrm{~N}^{*}$ state to the gas state. We chose the original reference state of CFF-AIMD to be the $2 \mathrm{~N}^{*}$ state because the PES at the $2 \mathrm{~N}^{*}$ minimum appears to be harmonic within thermal fluctuations $\left(k_{B} T \sim 0.06\right.$ to $0.12 \mathrm{eV}$ ) (see Figure S5C).

The procedure for changing the reference state was as follows. First, the lowestpotential energy configuration of the gas state was found from geometry optimization. The absolute free energy of the gas state was calculated based on the ideal gas approximation using the ASE thermochemistry module at pressure of 150 bar. Second, we used MCSHA, which showed best agreement with CFF-AIMD results among several HA method (see Figure 5 in the main text), to compute the absolute free energy of the $2 \mathrm{~N}^{*}$ state and, consequently, the free energy difference between the two reference states: $\Delta G_{g a s \rightarrow 2 N *}=$ $G($ gas $)-G(2 N *)$. Finally, we added the term $\Delta G_{g a s \rightarrow 2 N *}$ to the PMF computed from CFFAIMD, which was referenced to the $2 \mathrm{~N}^{*}$ state. To compute changes in enthalpy and entropy, we repeated the above steps with enthalpy (or entropy) in place of free energy.

\subsection{Recovering Unbiased Tilt Angle Distribution}

A weighting scheme was employed to recover unbiased distributions of physical quantities, such as the tilt angle, $\varnothing$, from biased simulations as in CFF. For each configuration sampled from CFF-AIMD simulations at temperature $T$, weights were assigned as follows:

$$
w(r, Z)=C e^{-G(r, Z) / k_{B} T}
$$

where $w(r, Z)$ is the weight, $G(r, Z)$ the free energy from CFF-AIMD, and $\mathrm{k}_{\mathrm{B}}$ the Boltzmann constant, and $C$ a normalization constant.

We calculated the distribution of the tilt angle for $\mathrm{N}_{2}$ by first selecting MD snapshots with CV values within a $0.02 \AA$ radius of those of the given state, and then constructing weighted histograms of $\varnothing$ for the selected configurations. The weight of each configuration was defined using Eq. S7. Finally, histograms were normalized to compute the probability density, $P(\varnothing)$.

\section{Adsorption Site Analysis}

An automated adsorption site identification algorithm was used to categorize nitrogen adsorption sites sampled from CFF-AIMD. The distribution of adsorption sites for the $2 \mathrm{~N}^{*}$ states was determined by selecting MD snapshots with $C V$ values $(r, Z)$ within a radius of $0.05 \AA$ of the $C V$ values of the free energy minimum for the $2 \mathrm{~N}^{*}$ state in the computed 2D-PMF: $(r, Z)=(2.70,1.09) \AA$ at $700 \mathrm{~K},(2.70,1.10) \AA$ at $1000 \mathrm{~K}$, and $(2.72$, 1.11) $\AA$ at $1400 \mathrm{~K}$. The adsorption sites on the Ru surface were identified as the face edges, face centers, and vertices of the Delaunay triangulation of the Ru surface atoms, as implemented in a Pymatgen module ${ }^{20,21}$. For a given nitrogen atom, its adsorption site was taken to be the closest one to the nitrogen atom in the in-plane distance ( $x$ and $y$ directions). The adsorption site was identified as one of the following: hollow, bridge, and on-top sites.

There are two possible hollow sites on an $\mathrm{HCP}(0001)$ facet of Ru surface: HCP or FCC. One of the main distinctions between HCP and FCC sites is that HCP sites are directly on top of an atom in the 2nd layer, whereas FCC sites are not. Therefore, to distinguish between an HCP and an FCC site, we use the shortest in-plane distance 
between the hollow site and an atom in the 2 nd layer. A cutoff distance of $0.6 \AA$ is able to classify HCP and FCC sites as shown in Figure S10.

For HCP-HCP and FCC-FCC configurations, there are two energetically nonequivalent configurations based on the in-plane angle $\theta\left(0^{\circ}\right.$ or $\left.60^{\circ}\right)$ defined in Figure S11A. This non-equivalence is due to the supercell configuration; the $0^{\circ}$ states have closer nearest-neighbor interactions than the $60^{\circ}$ in our model. This results in a total of five different $2 \mathrm{~N}^{*}$ states in our model, with $\mathrm{HCP}-\mathrm{HCP}$ configurations having lower energy than FCC-FCC configurations. Relative populations of dissociated states that are within the cutoff radius of the free energy minimum of $2 \mathrm{~N}^{*}$ are plotted in Figure S11B. Among the five states, only four of them (two HCP-HCP and two FCC-FCC configurations) fall within our selection criteria as expected based on their $(r, Z)$ values at the lowest-energy configuration found from energy minimization. These four states are used in MC-HA and MCS-HA calculations. 


\section{Supplementary Figures}
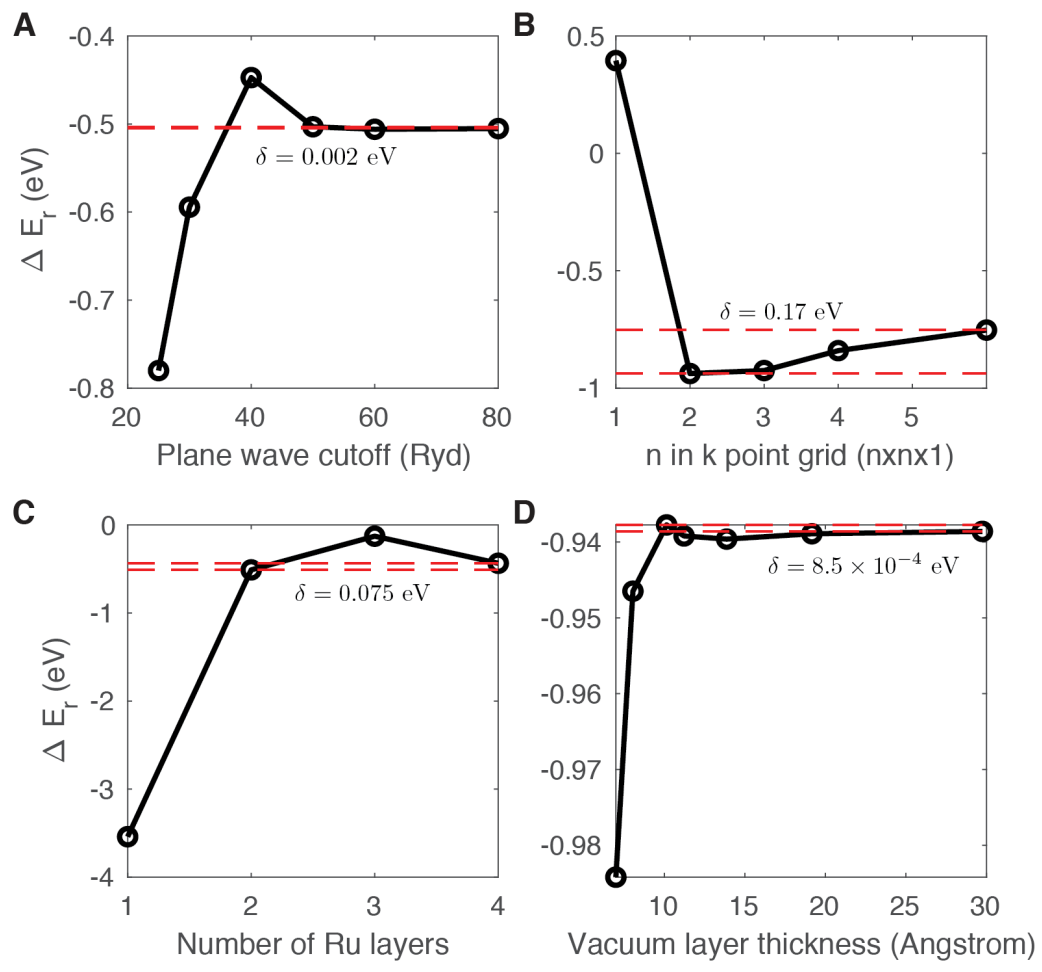

Figure S1: Convergence study of DFT parameters : (A) plane wave cutoff, (B) number of $k$ points, (C) number of Ru layers, and (D) thickness of the vacuum layer. The surface reaction energy, $\Delta E_{r}=E\left(\mathrm{~N}_{2}^{*}\right)-E\left(2 \mathrm{~N}^{*}\right)$, is plotted as a function of a varying DFT parameter. Unless told otherwise, the default parameters are as follows: a plane wave cutoff of 50 Ryd, a 2 X 2 X 1 k-point mesh, a two-layer Ru slab, and a vacuum layer thickness of $10.1 \AA$ (distance between periodic images of Ru slabs). The error associated with the chosen DFT parameter, $\delta$, is computed relative to its highest computed value, and is bounded by the two red dashed lines. 

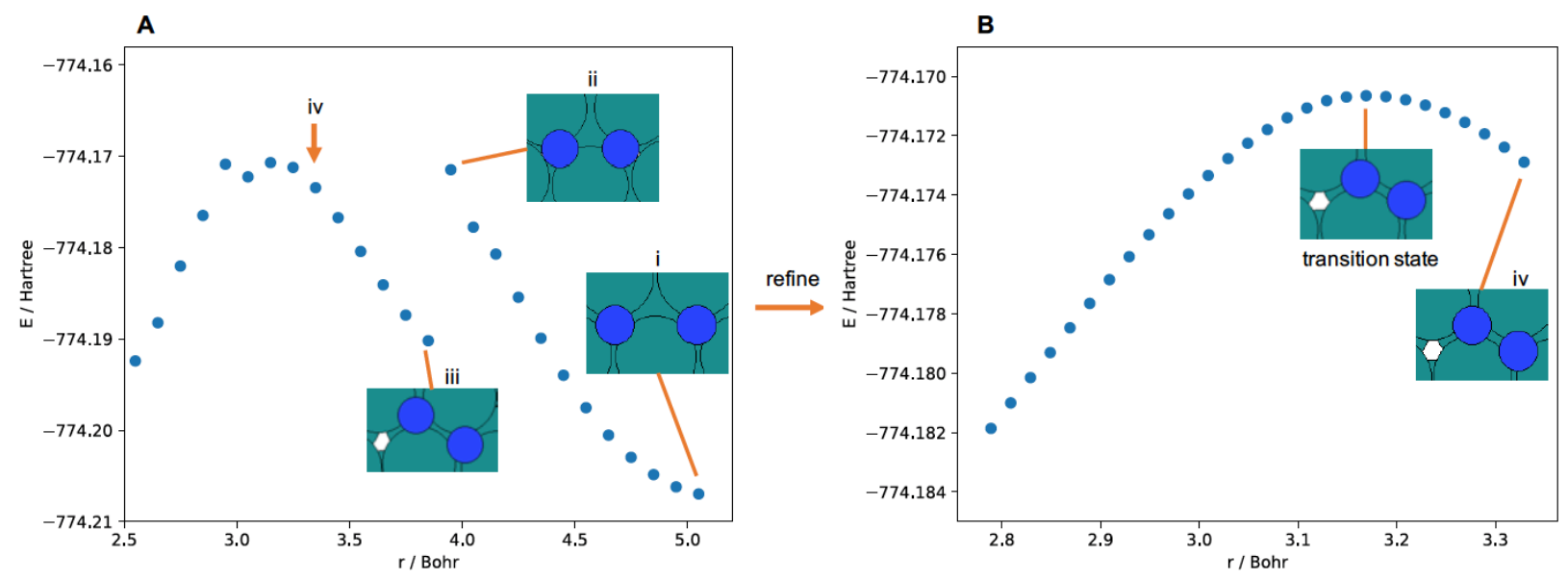

Figure S2: Transition state search from an FCC-FCC starting configuration. (A) Initial FBL calculation with a bond-length constraint step size of 0.1 Bohr. (B) Final FBL calculation with a smaller step size of 0.02 Bohr. Configuration (i) is the starting configuration. The jump in energy from configurations (ii) to (iii) represents a diffusion barrier (from an FCC site to a neighboring HCP site), which is irrelevant for calculating the reaction barrier. Configuration (iv) is taken for a refined transition state search as shown in (B), resulting in the final converged transition state for the FCC-FCC starting configuration search.
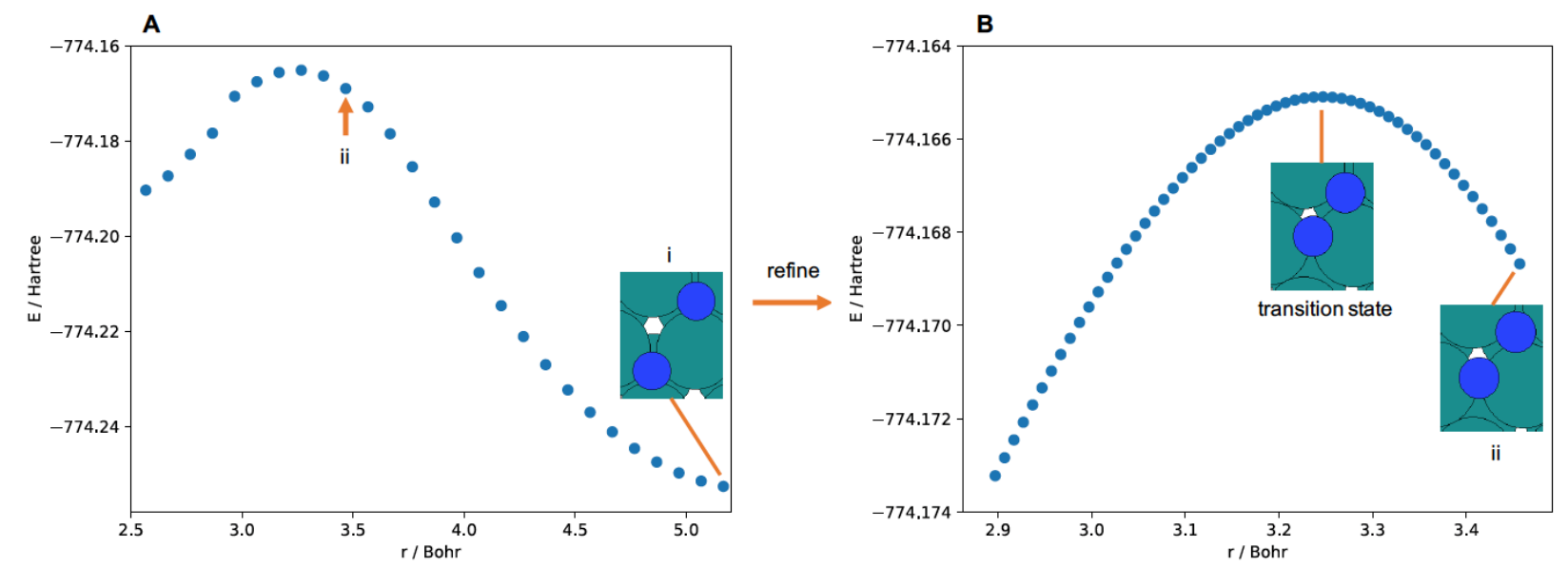

Figure S3: Transition state search from an HCP-HCP starting configuration. (A) Initial FBL calculation with a bond-length constraint step size of 0.1 Bohr. (B) Final FBL calculation with a smaller step size of 0.01 Bohr. Configuration (i) is the starting configuration. Configuration (iv) is taken for a refined transition state search as shown in $(B)$, resulting in the final converged transition state for the FCC-FCC starting configuration search. 

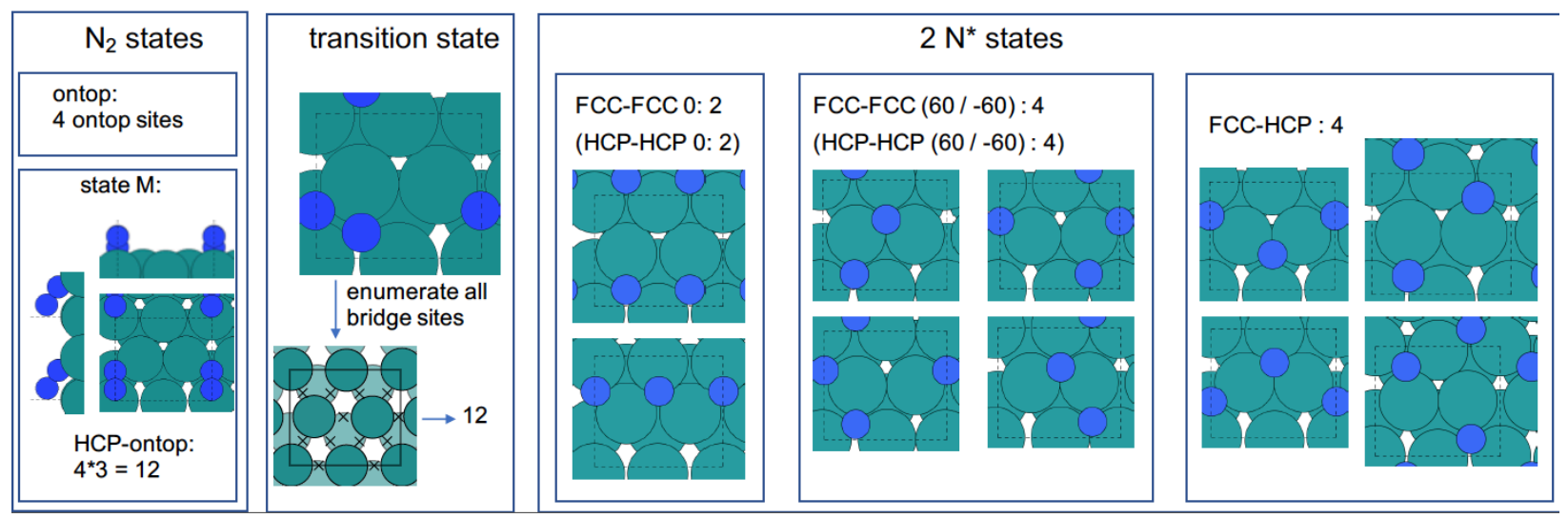

Figure S4: Degeneracies or configurational multiplicities of $\mathrm{N}_{2}{ }^{*}, \mathrm{TS}$, and $2 \mathrm{~N}^{*}$ states. The configurations are found by energy minimization $\left(\mathrm{N}_{2}{ }^{*}\right.$ and $2 \mathrm{~N}^{*}$ states) and $\mathrm{FBL}$ calculations (TS).
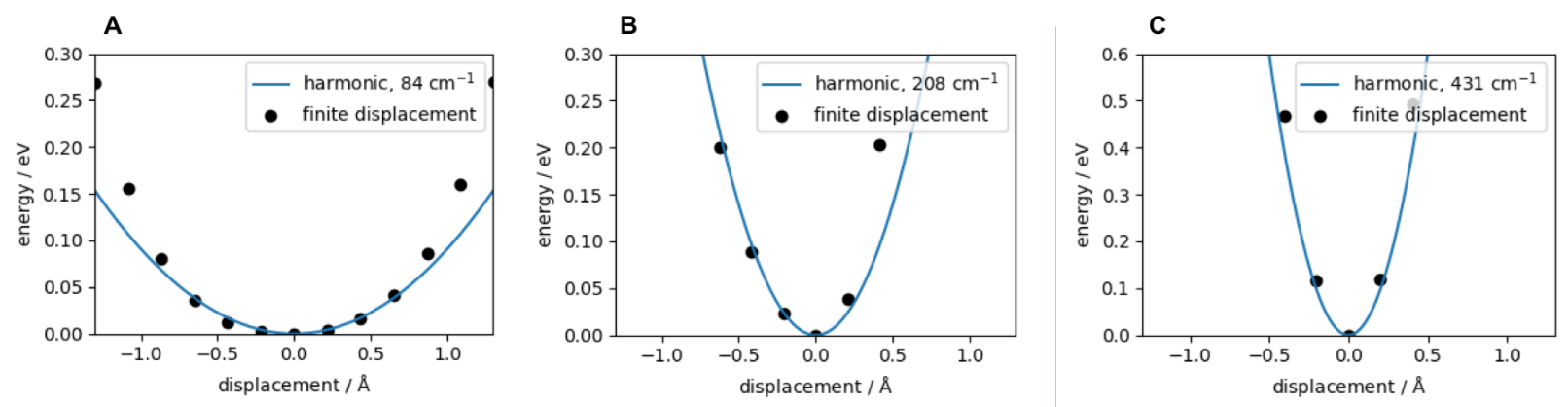

Figure S5: Comparison of harmonic approximation theory (solid lines) and single-point calculations (points) of the potential energy surface along the eigenvector corresponding to the lowest eigenvalue for (A) $\mathrm{N}_{2}{ }^{*}$, (B) TS, and (C) $2 \mathrm{~N}^{*}\left(\mathrm{HCP}-\mathrm{HCP} 60^{\circ}\right)$ states. Energy is referenced to the value at zero displacement. 


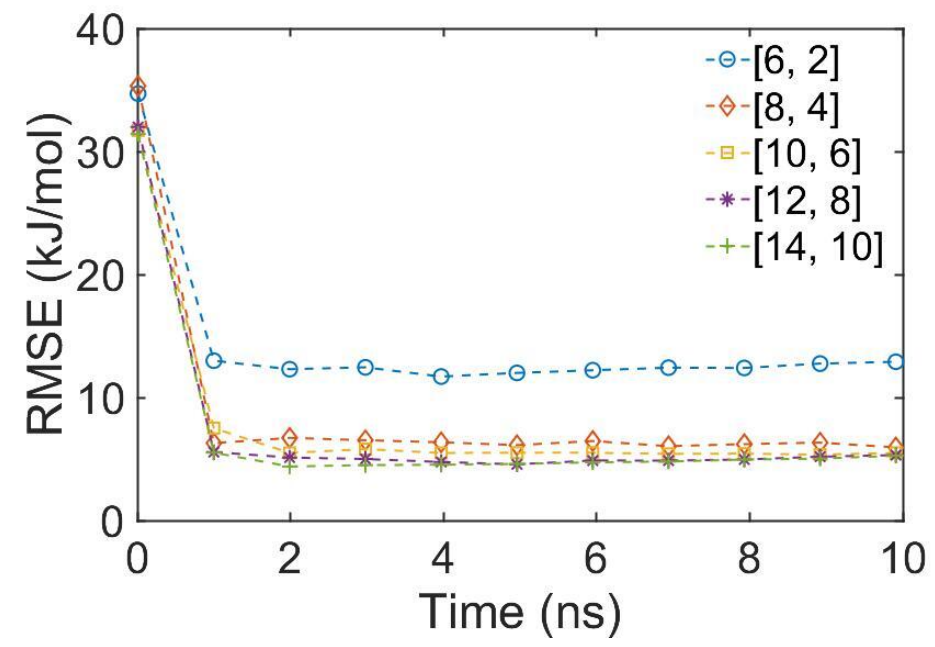

Figure S6: Root mean squared error (RMSE) in PMF for alanine dipeptide using various BRANN architectures. The RMSE is computed relative to a reference PMF obtained from a 100 ns-long simulation using ABF. The performance of BRANN appears to be insensitive to neural network sizes of $[8,4]$ and larger.
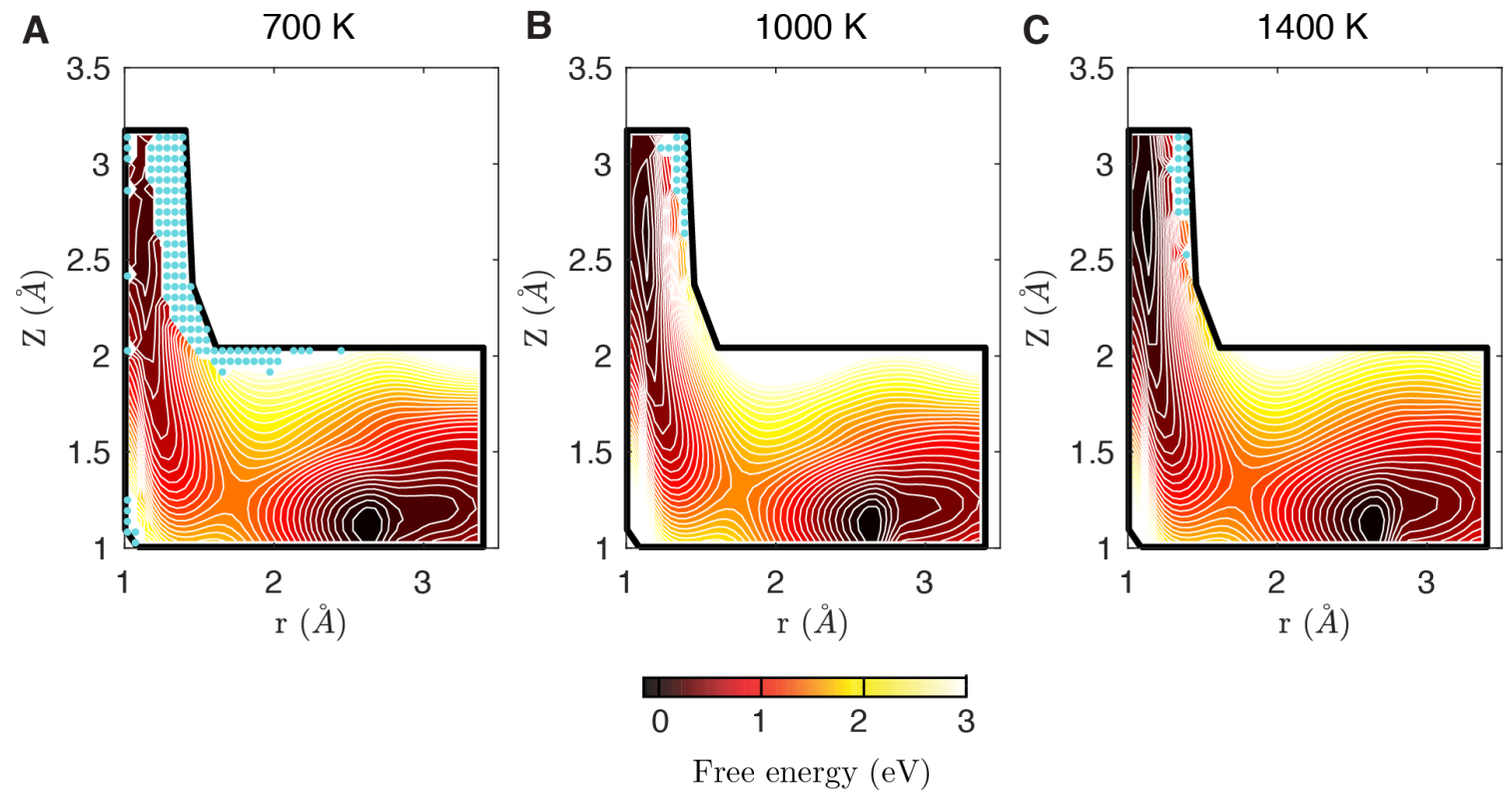

Figure S7: 2D-PMFs from ABF-AIMD simulations (A) at $700 \mathrm{~K}$ for $71 \mathrm{ps},(\mathrm{B})$ at $1000 \mathrm{~K}$ after 70 ps, and (C) $1400 \mathrm{~K}$ after $102 \mathrm{ps}$, using 14 to 20 walkers. Same 2D discretized CV grid was used as in CFF-AIMD simulations. 2D-PMFs were calculated by integrating mean forces over the sampled CV grid points that fall within the non-rectangular $2 \mathrm{D}$ boundary (delineated by black lines). Blue circles indicate the unsampled CV grid points, which are concentrated near the $\mathrm{N}_{2}{ }^{*}$ state. 

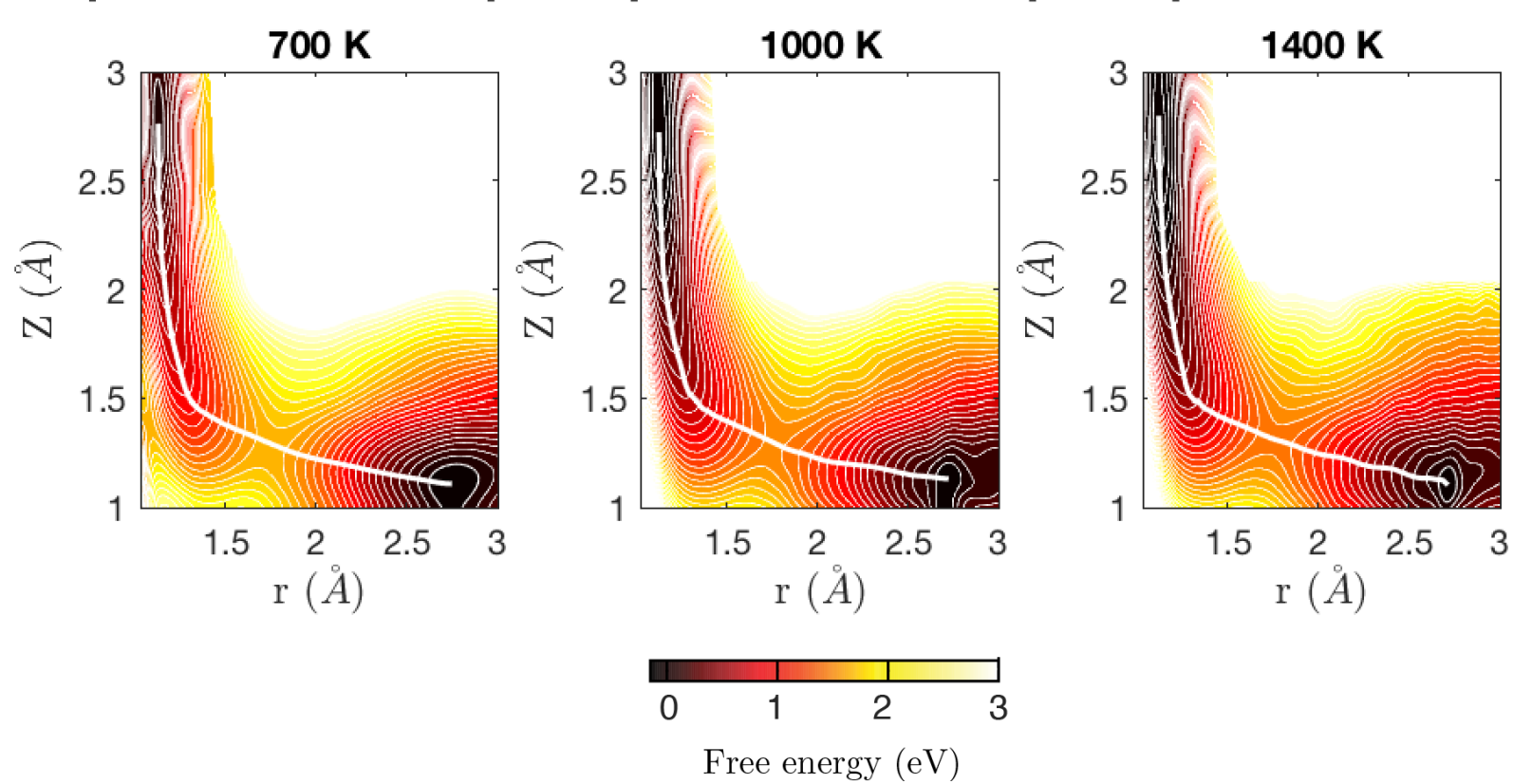

Figure S8: Averaged 2D-PMFs from CFF-AIMD simulations at $700 \mathrm{~K}, 1000 \mathrm{~K}$, and 1400 $\mathrm{K}$. The MFEPs connecting $\mathrm{N}_{2}{ }^{*}$ and $2 \mathrm{~N}^{*}$ minima are marked using white solid lines.

A

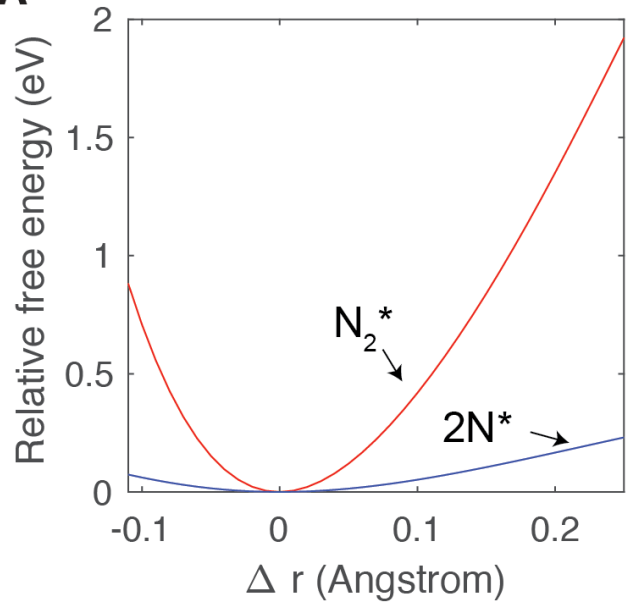

B

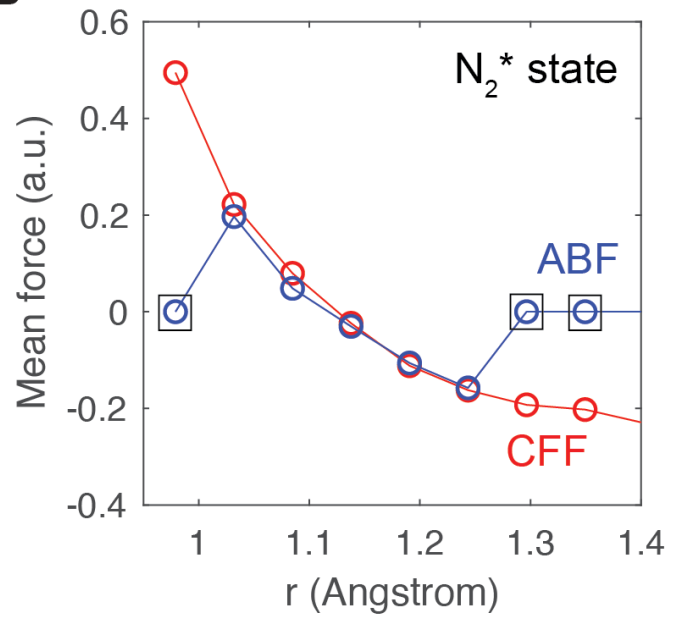

Figure S9: Free energy and its gradient of the $\mathrm{N}_{2}{ }^{*}$ state at $1400 \mathrm{~K}$. (A) Changes in free energy of $\mathrm{N}_{2}{ }^{*}$ (at $Z=2.7 \AA$ ) and $2 \mathrm{~N}^{*}$ (at $Z=1.1 \AA$ ) states near their respective minima along the r-direction. Free energies were computed from a converged CFF-AIMD simulation. The $\mathrm{N}_{2}{ }^{*}$ state has a deeper and narrower energy well than that of the $2 \mathrm{~N}^{*}$ state. (B) Gradient of the free energy (i.e., mean forces) in the $\mathrm{N}_{2}{ }^{*}$ region (at $Z=2.7 \AA$ ) from CFFAIMD and ABF-AIMD simulations (same data plotted in Figures $2 C$ and $2 F$ in the main text). The mean force profile (negative of biasing forces) from the ABF method shows discontinuities along the r-direction away from the $\mathrm{N}_{2}{ }^{*}$ minimum as the energy changes rapidly. ABF data points marked in black squares indicate unsampled regions; therefore, the biasing forces are zero in those regions. 

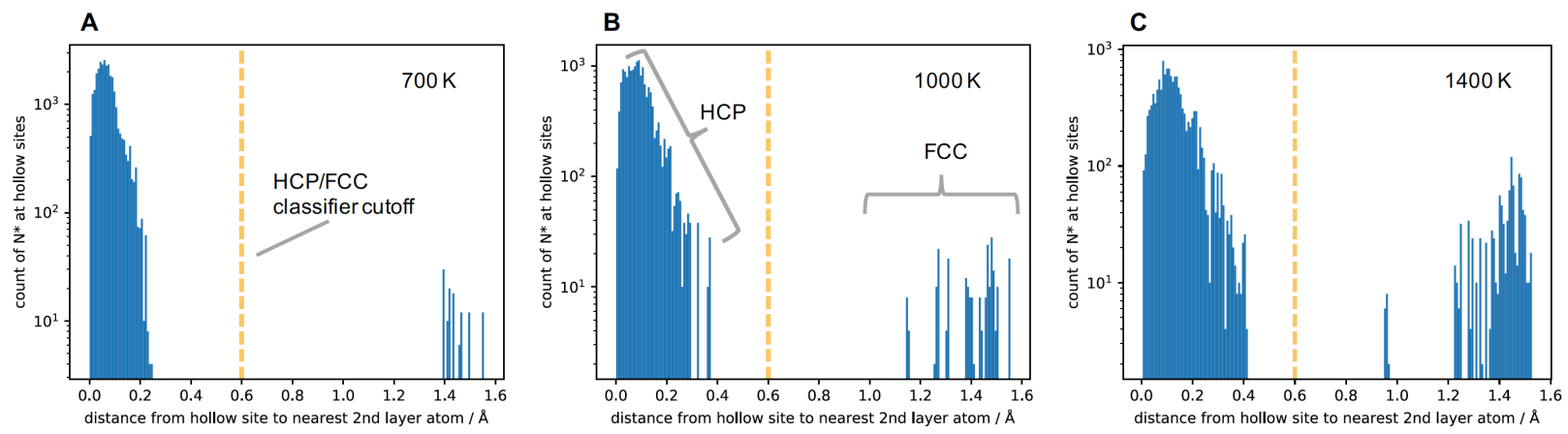

Figure S10: FCC/HCP hollow site classification using MD snapshots from CFF-AIMD simulations at (A) $700 \mathrm{~K},(\mathrm{~B}) 1000 \mathrm{~K}$, and (C) $1400 \mathrm{~K}$.

A

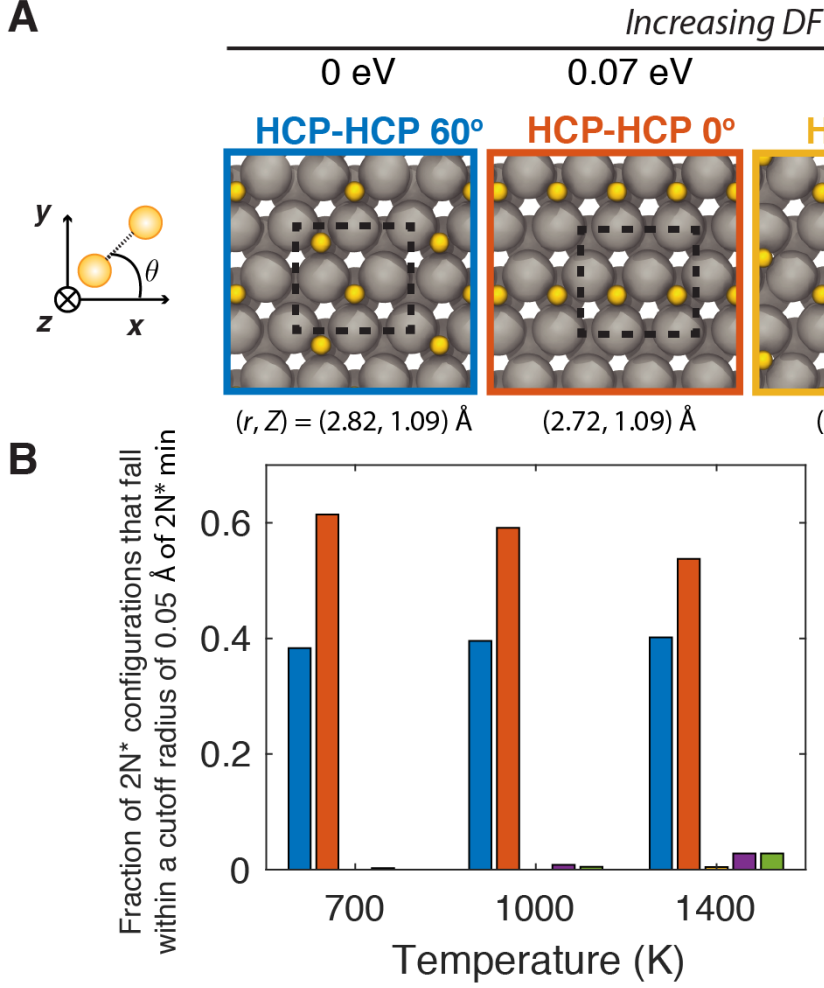

ing DFT energy of $2 N^{*}$ states

$0.56 \mathrm{eV}$

$1.09 \mathrm{eV}$

$1.25 \mathrm{eV}$

HCP-FCC

FCC-FCC $60^{\circ}$

FCC-FCC $0^{\circ}$

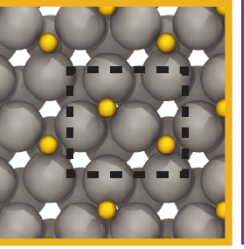

$(3.22,1.18) \AA$

$(2.72,1.02) \AA$
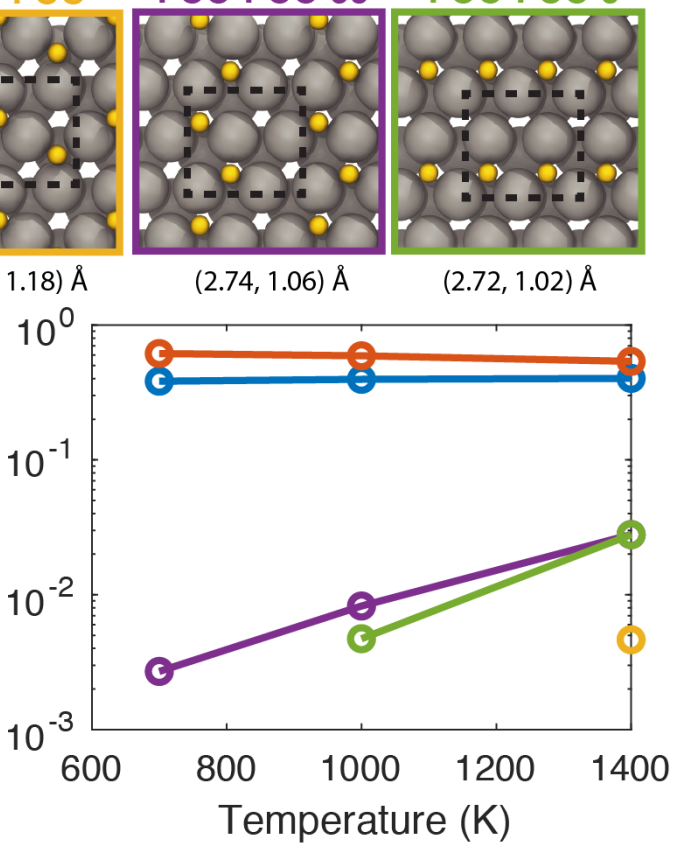

Figure S11: Analysis of dissociated states. (A) Relative DFT energies of $2 \mathrm{~N}^{*}$ local minima. The lowest-energy configurations shown here are found using energy minimization. Energies are calculated relative to the $\mathrm{HCP}-\mathrm{HCP} 60^{\circ}$ configuration. The $\mathrm{CV}$ values of their configurations are shown below each structure. Except HCP-FCC, other minima fall within a cutoff radius of $0.05 \AA$ of the $2 \mathrm{~N}^{*}$ free energy minimum in the CV-space, $(r, Z) \sim$ $(2.7,1.1) \AA$. (B) Fraction of $2 \mathrm{~N}^{*}$ configurations that are within the cutoff radius of the $2 \mathrm{~N}^{*}$ free energy minimum in the CV-space. The left panel is plotted on a linear scale, whereas the right panel is on a log scale. Unbiased distributions are computed from biased simulation trajectories by applying a weighted histogram scheme with weights defined in Eq. S7. 


\section{Supplementary Tables}

Table S1: Comparisons of free energies of reaction, $\Delta G_{r}$, for $N_{2}{ }^{*} \rightarrow 2 N^{*}$ and activation energies, $\Delta G^{\neq}=G(T S)-G\left(2 N^{*}\right)$, for classical and quantum harmonic oscillator models. Here we use a single lowest-energy configuration for each critical points $\left(\mathrm{N}_{2}{ }^{*}, \mathrm{TS}\right.$, and $2 \mathrm{~N}^{*}$ ) of the PES as done in SC-HA, but with either classical or quantum harmonic oscillator models.

\begin{tabular}{lcccc}
\hline \multicolumn{1}{c}{$\mathrm{T}(\mathrm{K})$} & $\begin{array}{c}\text { classical } \\
\Delta G_{r} \\
(\mathrm{eV})\end{array}$ & $\begin{array}{c}\text { quantum } \\
\Delta G_{r} \\
(\mathrm{eV})\end{array}$ & $\begin{array}{c}\text { classical } \\
\Delta G^{\neq} \\
(\mathrm{eV})\end{array}$ & $\begin{array}{c}\text { quantum } \\
\Delta G^{\neq} \\
(\mathrm{eV})\end{array}$ \\
\hline 100 & -0.47 & -0.51 & 2.22 & 2.17 \\
300 & -0.42 & -0.46 & 2.18 & 2.16 \\
700 & -0.30 & -0.33 & 2.14 & 2.13 \\
1000 & -0.22 & -0.24 & 2.12 & 2.12 \\
1400 & -0.10 & -0.12 & 2.12 & 2.11 \\
\hline
\end{tabular}

Table S2: Summary of CFF-AIMD simulations. A total of $\sim 500$ ps of simulations were performed using multiple walkers, amounting to $\sim 1.7$ million timesteps.

\begin{tabular}{|l|l|l|l|l|}
\hline \multirow{3}{*}{$T(\mathrm{~K})$} & \multicolumn{1}{|c|}{ Trial } & \multicolumn{1}{|c|}{$\begin{array}{c}\text { Number of } \\
\text { walkers }\end{array}$} & $\begin{array}{c}\text { Total } \\
\text { simulation time } \\
(\mathrm{ps})\end{array}$ & $\begin{array}{c}\text { Total number } \\
\text { of timesteps } \\
\text { using multiple } \\
\text { walkers }\end{array}$ \\
\hline \multirow{3}{*}{700} & 1 & avg. 17 & 99.6 & 3654070 \\
\cline { 2 - 6 } & 2 & avg. 21 & 33.1 & 1021770 \\
\cline { 2 - 6 } & 3 & avg. 24 & 25.5 & 1254216 \\
\hline \multirow{3}{*}{1000} & 1 & avg. 17 & 96.4 & 3322857 \\
\cline { 2 - 6 } & 2 & avg. 15 & 30.0 & 697601 \\
\cline { 2 - 6 } & 3 & avg. 14 & 23.4 & 878403 \\
\hline \multirow{3}{*}{1400} & 1 & avg. 14 & 113.6 & 3010901 \\
\cline { 2 - 6 } & 2 & 16 & 49.3 & 1104000 \\
\cline { 2 - 6 } & 3 & 16 & 35.3 & 1632000 \\
\hline
\end{tabular}




\section{References}

(1) Perdew, J. P.; Burke, K.; Ernzerhof, M. Generalized Gradient Approximation Made Simple. Phys. Rev. Lett. 1996, 77 (18), 3865-3868.

(2) Schlipf, M.; Gygi, F. Optimization Algorithm for the Generation of ONCV Pseudopotentials. Comput. Phys. Commun. 2015, 196, 36-44.

(3) Hamann, D. R. Optimized Norm-Conserving Vanderbilt Pseudopotentials. Phys. Rev. B 2013, 88 (8), 085117.

(4) Henkelman, G.; Uberuaga, B. P.; Jónsson, H. A Climbing Image Nudged Elastic Band Method for Finding Saddle Points and Minimum Energy Paths. J. Chem. Phys. 2000, 113 (22), 9901-9904.

(5) Alavi, A.; Hu, P.; Deutsch, T.; Silvestrelli, P. L.; Hutter, J. CO Oxidation on Pt(111): An Ab Initio Density Functional Theory Study. Phys. Rev. Lett. 1998, 80 (16), 36503653.

(6) Giannozzi, P.; Baroni, S.; Bonini, N.; Calandra, M.; Car, R.; Cavazzoni, C.; Ceresoli, D.; Chiarotti, G. L.; Cococcioni, M.; Dabo, I.; Dal Corso, A.; de Gironcoli, S.; Fabris, S.; Fratesi, G.; Gebauer, R.; Gerstmann, U.; Gougoussis, C.; Kokalj, A.; Lazzeri, M.; Martin-Samos, L.; Marzari, N.; Mauri, F.; Mazzarello, R.; Paolini, S.; Pasquarello, A.; Paulatto, L.; Sbraccia, C.; Scandolo, S.; Sclauzero, G.; Seitsonen, A. P.; Smogunov, A.; Umari, P.; Wentzcovitch, R. M. QUANTUM ESPRESSO: A Modular and Open-Source Software Project for Quantum Simulations of Materials. J. Phys.: Condens. Matter 2009, 21 (39), 395502.

(7) Hjorth Larsen, A.; Jørgen Mortensen, J.; Blomqvist, J.; Castelli, I. E.; Christensen, R.; Dułak, M.; Friis, J.; Groves, M. N.; Hammer, B.; Hargus, C.; Hermes, E. D.; Jennings, P. C.; Bjerre Jensen, P.; Kermode, J.; Kitchin, J. R.; Leonhard Kolsbjerg, E.; Kubal, J.; Kaasbjerg, K.; Lysgaard, S.; Bergmann Maronsson, J.; Maxson, T.; Olsen, T.; Pastewka, L.; Peterson, A.; Rostgaard, C.; Schiøtz, J.; Schütt, O.; Strange, M.; Thygesen, K. S.; Vegge, T.; Vilhelmsen, L.; Walter, M.; Zeng, Z.; Jacobsen, K. W. The Atomic Simulation Environment-a Python Library for Working with Atoms. J. Phys. Condens. Matter 2017, 29 (27), 273002.

(8) Gygi, F. Qbox Utility Code http://scherzo.ucdavis.edu/qbox/qboxpublic/tree/master/util/dynmat (accessed Oct 17, 2019).

(9) Shakouri, K.; Behler, J.; Meyer, J.; Kroes, G.-J. Accurate Neural Network Description of Surface Phonons in Reactive Gas-Surface Dynamics: $\mathrm{N}_{2}+\mathrm{Ru}(0001)$. J. Phys. Chem. Lett. 2017, 8 (10), 2131-2136.

(10) Sevgen, E.; Guo, A. Z.; Sidky, H.; Whitmer, J. K.; de Pablo, J. J. Combined ForceFrequency Sampling for Simulation of Systems Having Rugged Free Energy Landscapes. J. Chem. Theory Comput. 2020, 16 (3), 1448-1455.

(11) Sidky, H.; Colón, Y. J.; Helfferich, J.; Sikora, B. J.; Bezik, C.; Chu, W.; Giberti, F.; Guo, A. Z.; Jiang, X.; Lequieu, J.; Li, J.; Moller, J.; Quevillon, M. J.; Rahimi, M.; Ramezani-Dakhel, H.; Rathee, V. S.; Reid, D. R.; Sevgen, E.; Thapar, V.; Webb, M. A.; Whitmer, J. K.; de Pablo, J. J. SSAGES: Software Suite for Advanced General Ensemble Simulations. J. Chem. Phys. 2018, 148 (4), 044104.

(12) Darve, E.; Rodríguez-Gómez, D.; Pohorille, A. Adaptive Biasing Force Method for Scalar and Vector Free Energy Calculations. J. Chem. Phys. 2008, 128 (14), 144120. 
(13) Guo, A. Z.; Sevgen, E.; Sidky, H.; Whitmer, J. K.; Hubbell, J. A.; de Pablo, J. J. Adaptive Enhanced Sampling by Force-Biasing Using Neural Networks. J. Chem. Phys. 2018, 148 (13), 134108.

(14) Sidky, H.; Whitmer, J. K. Learning Free Energy Landscapes Using Artificial Neural Networks. J. Chem. Phys. 2018, 148 (10), 104111.

(15) Abraham, M. J.; Murtola, T.; Schulz, R.; Páll, S.; Smith, J. C.; Hess, B.; Lindahl, E. GROMACS: High Performance Molecular Simulations through Multi-Level Parallelism from Laptops to Supercomputers. SoftwareX 2015, 1-2, 19-25.

(16) Hornak, V.; Abel, R.; Okur, A.; Strockbine, B.; Roitberg, A.; Simmerling, C. Comparison of Multiple Amber Force Fields and Development of Improved Protein Backbone Parameters. Proteins 2006, 65 (3), 712-725.

(17) Jorgensen, W. L.; Chandrasekhar, J.; Madura, J. D.; Impey, R. W.; Klein, M. L. Comparison of Simple Potential Functions for Simulating Liquid Water. J. Chem. Phys. 1998, 79 (2), 926.

(18) Parrinello, M.; Rahman, A. Polymorphic Transitions in Single Crystals: A New Molecular Dynamics Method. J. Appl. Phys. 1981, 52 (12), 7182-7190.

(19) Henkelman, G.; Jónsson, H. Improved Tangent Estimate in the Nudged Elastic Band Method for Finding Minimum Energy Paths and Saddle Points. J. Chem. Phys. 2000, 113 (22), 9978-9985.

(20) Ong, S. P.; Richards, W. D.; Jain, A.; Hautier, G.; Kocher, M.; Cholia, S.; Gunter, D.; Chevrier, V. L.; Persson, K. A.; Ceder, G. Python Materials Genomics (Pymatgen): A Robust, Open-Source Python Library for Materials Analysis. Comput. Mater. Sci. 2013, 68, 314-319.

(21) Montoya, J. H.; Persson, K. A. A High-Throughput Framework for Determining Adsorption Energies on Solid Surfaces. npj Comput. Mater. 2017, 3 (1), 1-4. 